International Electronic Journal of Geometry

Volume 7 No. 2 PP. $72-83$ (2014) (CIEJG

\title{
MULTIPLY WARPED PRODUCT SUBMANIFOLDS OF A GENERALIZED SASAKIAN SPACE FORM
}

\author{
MOHAMMED JAMALI AND MOHAMMAD HASAN SHAHID \\ (Communicated by Cihan ÖZGÜR)
}

\begin{abstract}
In this article, we derive some inequalities for second fundamental form and warping functions for multiply warped product submanifolds of a generalized Sasakian space form.
\end{abstract}

\section{INTRODUCTION}

It is known that the notion of CR-warped product manifold was first introduced by B. Y. Chen([3],[4]). In these papers he obtained the certain sharp inequalities involving warping functions and the squared norm of second fundamental form. After that B. Sahin [11] extended the Chen's result for warped product semi-slant submanifolds of Kaehler manifold. Later I. Mihai [7] improved the same type of inequality in case of contact CR-warped products in Sasakian space form. Moreover Marian-Ioan Munteanu [8] also studied warped product contact CR-submanifolds of Sasakian space form. Some non-existence results were also obtained by M. Atceken [2] for certain class of submanifolds of a Kenmotsu manifold. In the present article we will calculate the inequalities of same kind for different types of multiply warped product submanifolds of generalized Sasakian space form.

Let $N_{1}, N_{2}, \ldots . ., N_{k}$ be Riemannian manifolds and let $N=N_{1} \times N_{2} \times \ldots \times N_{k}$ be the cartesian product of $N_{1}, N_{2}, \ldots, N_{k}$. For each $a$, denote by $\pi_{a}: N \longrightarrow N_{a}$ the canonical projection of $N$ onto $N_{a}$. We denote the horizontal lift of $N_{a}$ in $N$ via $\pi_{a}$ by $N_{a}$ itself.

If $\sigma_{2}, \ldots, \sigma_{k}: N_{1} \longrightarrow R^{+}$are positive valued functions, then

$$
\langle X, Y\rangle=\left\langle\pi_{*} X, \pi_{*} Y\right\rangle+\sum_{a=1}^{k}\left(\sigma_{a} \circ \pi_{1}\right)^{2}\left\langle\pi_{a *} X, \pi_{a *} Y\right\rangle
$$

defines a Riemannian metric $g$ on $N$, called a multiply warped product metric. The product manifold $N$ endowed with this metric is denoted by $N_{1} \times_{\sigma_{2}} N_{2} \times \ldots \times{ }_{\sigma_{k}} N_{k}$.

For a multiply warped product manifold $N_{1} \times{ }_{\sigma_{2}} N_{2} \times \ldots \times \times_{\sigma_{k}} N_{k}$, let $D_{a}$ denote the distributions obtained from the vectors tangent to $N_{a}$.

Date: Received: December 2, 2012, Accepted: October 9, 2013.

2010 Mathematics Subject Classification. 53C40, 53C25.

Key words and phrases. Multiply warped product, Generalized Sasakian space form. 
Let $x: N_{1} \times_{\sigma_{2}} N_{2} \times \ldots \times_{\sigma_{k}} N_{k} \longrightarrow \bar{M}$ be an isometric immersion of of a multiply warped product $N_{1} \times_{\sigma_{2}} N_{2} \times \ldots \times_{\sigma_{k}} N_{k}$ into a Riemannian manifold $\bar{M}$. Denote by $h$ the second fundamental form of $x$. Then the immersion $x$ is called mixed totally geodesic if $h\left(D_{a}, D_{b}\right)=0$ holds for distinct $a, b \in\{1,2, \ldots, k\}$.

Denote by trace $h_{a}$ the trace of $h$ restricted to $N_{a}$, that is

$$
\text { trace } h_{a}=\sum_{\alpha=1}^{n_{a}} h\left(e_{\alpha}, e_{\alpha}\right)
$$

for some orthonormal frame fields $e_{1}, e_{2}, \ldots, e_{n_{a}}$ of $D_{a}$.

A submanifold $M$ of an almost contact manifold $\bar{M}$ is called invariant if the almost contact structure $\phi$ of $\bar{M}$ carries each tangent space of $M$ into itself whereas it is said to be anti-invariant if the almost contact structure $\phi$ of $\bar{M}$ carries each tangent space of $M$ into its corresponding normal space.

A multiply warped product $N_{T} \times_{\sigma_{2}} N_{2} \times \ldots \times_{\sigma_{k}} N_{k}$ in an almost contact metric manifold $\bar{M}$ is called a multiply CR-warped product if $N_{T}$ is an invariant submanifold and $N_{\perp}={ }_{\sigma_{2}} N_{2} \times \ldots \times{ }_{\sigma_{k}} N_{k}$ is an anti-invariant submanifold of $\bar{M}$.

In [6] Chen and Dillen obtained the following inequality for warping functions:

Theorem 1.1. Let $x: N_{1} \times_{\sigma_{2}} N_{2} \times \ldots \times{ }_{\sigma_{k}} N_{k} \longrightarrow \bar{M}^{m}$ be an isometric immersion of a multiply warped product $N^{n}=N_{1} \times_{\sigma_{2}} N_{2} \times \ldots \times{ }_{\sigma_{k}} N_{k}$ into an arbitrary Riemannian manifold $\bar{M}$. Then we have

$$
\sum_{j=2}^{k} n_{j} \frac{\Delta \sigma_{j}}{\sigma_{j}} \leq \frac{n^{2}}{4}\|H\|^{2}+n_{1}\left(n-n_{1}\right) \max \bar{K}, \quad n=\sum_{j=1}^{k} n_{j},
$$

where $\max \bar{K}(p)$ denotes the maximum of the sectional curvature function of $\bar{M}^{m}$ restricted to 2-planes sections of the tangent space $T_{p} N$ of $N$ at $p=\left(p_{1}, \ldots, p_{k}\right)$.

The equality holds identically if and only if the following two statements hold:

(1) $x$ is a mixed totally geodesic immersion satisfying trace $h_{1}=\ldots . .=$ trace $h_{k}$

(2) at each point $p \in N$, the sectional curvature function $\bar{K}$ of $\bar{M}$ satisfies $\bar{K}(u, v)=\max \bar{K}(p)$ for each unit vector $u$ in $T_{p_{1}}\left(N_{1}\right)$ and each unit vector $v$ in $T_{\left(p_{2}, \ldots, p_{k}\right)}\left(N_{2} \times \ldots \times N_{k}\right)$.

They also derived inequality between the squared norm of the second fundamental form $h$ and the gradient of warping functions.

Recently, in [9][10] A. Olteanu achieved the similar results in case of multiply CR-warped product submanifolds of a Kenmotsu space form and doubly warped product submanifolds of generalized Sasakian space form. In the present article we obtain the similar inequalities for multiply CR-warped product submanifolds of a generalized Sasakian space form.

\section{Preliminaries}

A $(2 m+1)$-dimensional Riemannian manifold $\bar{M}$ is said to be an almost contact metric manifold if it admits an endomorphism $\phi$, a vector field $\xi$, a 1-form $\eta$ and a Riemannian metric $g$ satisfying the following properties:

$$
\begin{gathered}
\phi^{2}=-I d+\eta \otimes \xi, \quad \eta(\xi)=1, \quad \phi \xi=0, \quad \eta \circ \phi=0, \\
g(\phi X, \phi Y)=g(X, Y)-\eta(X) \eta(Y), \quad \eta(X)=g(X, \xi),
\end{gathered}
$$


for any vector fields $X, Y$ on $\bar{M}$.

$$
g(X, \phi Y)=-g(\phi X, Y)
$$

A plane section $\pi$ in $T_{p} \bar{M}$ is called a $\phi$-section if it is spanned by $X$ and $\phi X$, where $X$ is a unit tangent vector orthogonal to $\xi$. The sectional curvature of a $\phi$-section is called a $\phi$-sectional curvature. A Sasakian (resp. Kenmotsu and cosymplectic) manifold with constant $\phi$-sectional curvature is a Sasakian (resp. Kenmotsu and cosymplectic) space form. In 2004 [1] Alegre, Blair and Carriazo introduced a space form called generalized Sasakian space form which generalized Sasakian, Kenmotsu and cosymplectic space forms.

An almost contact metric manifold $\bar{M}(\phi, \xi, \eta, g)$ is said to be generalized Sasakian space form if there exist three functions $f_{1}, f_{2}$ and $f_{3}$ on $\bar{M}$ such that the curvature tensor $\bar{R}$ is given by [1]

$$
\begin{gathered}
\bar{R}(X, Y) Z=f_{1}\{g(Y, Z) X-g(X, Z) Y\} \\
+f_{2}\{g(X, \phi Z) \phi Y-g(Y, \phi Z) \phi X+2 g(X, \phi Y) \phi Z\} \\
+f_{3}\{\eta(X) \eta(Z) Y-\eta(Y) \eta(Z) X+g(X, Z) \eta(Y) \xi-g(Y, Z) \eta(X) \xi\} .
\end{gathered}
$$

Let $\bar{\nabla}$ denote the connection on generalized Sasakian space form $\bar{M}$. If $M$ is a submanifold of a generalized Sasakian space form $\bar{M}$, we denote the induced metric on $M$ by the same symbol $g$ whereas the induced connection on $M$ by $\nabla$. The Gauss and Weingarten formulae are given by

$$
\begin{aligned}
& \bar{\nabla}_{X} Y=\nabla_{X} Y+h(X, Y), \\
& \bar{\nabla}_{X} N=-A_{N} X+\nabla_{X}^{\perp} N
\end{aligned}
$$

for each $X, Y \in T M$ and $N \in T^{\perp} M$, where $\nabla^{\perp}$ denotes the induced connection on the normal bundle $T^{\perp} M, h$ and $A_{N}$ are the second fundamental form and the shape operator of the immersion of $M$ into $\bar{M}$. The relation between $h$ and $A_{N}$ is given as

$$
g(h(X, Y), N)=g\left(A_{N} X, Y\right) .
$$

The covariant derivative of the second fundamental form $h$ is defined as

$$
\left(\bar{\nabla}_{X} h\right)(Y, Z)=\nabla^{\perp} h(Y, Z)-h\left(\nabla_{X} Y, Z\right)-h\left(Y, \nabla_{X} Z\right)
$$

for any $X, Y, Z \in T M$.If $\bar{R}$ and $R$ are the curvature tensors of the connections $\bar{\nabla}$ and $\nabla$ on $\bar{M}$ and $M$ respectively, the Gauss and Codazzi equation are mentioned as

$$
\begin{gathered}
\bar{R}(X, Y, Z, W)=R(X, Y, Z, W)-g(h(X, Z), g(Y, W))+g(h(X, W), h(Y, Z)) \\
(\bar{R}(X, Y) Z)^{\perp}=\left(\bar{\nabla}_{X} h\right)(Y, Z)-\left(\bar{\nabla}_{Y} h\right)(X, Z),
\end{gathered}
$$

respectively.

Let $N=N_{T} \times{ }_{\sigma_{2}} N_{2} \times \ldots \times{ }_{\sigma_{k}} N_{k}$ be a multiply $C R$-warped product submanifold of a generalized Sasakian space form. Let $D$ be the invariant distribution (i.e. $T N_{T}=$ $D)$ such that its orthogonal complementary distribution $D^{\perp}$ is anti-invariant (i.e. $\left.T N_{\perp}=T\left(N_{2} \times \ldots \times N_{k}\right)=D^{\perp}\right)$. We take $\{\xi\}$ to be tangent to $T N$. Then we have the following decompositions

$$
T N=D \oplus D^{\perp} \oplus\{\xi\},
$$




$$
T^{\perp} N=\phi D^{\perp} \oplus \lambda
$$

where $\lambda$ denotes the orthogonal complementary distribution of $\phi D^{\perp}$ and is an invariant normal subbundle of $T^{\perp} N$.

For any vector field $X \in T N$ we put

$$
\left(\bar{\nabla}_{X} \phi\right) Y=P_{X} Y+Q_{X} Y
$$

where $P X$ (resp. $Q X)$ denotes the tangential (resp. normal) component of $\phi X$. Also let $p \in N$ and $\left\{e_{1}, \ldots, e_{n}, e_{n+1}, \ldots, e_{2 m+1}\right\}$ an orthonormal basis of the tangent space $T_{p} \bar{M}$, such that $e_{1}, \ldots e_{n}$ are tangents to $N$ at $p$ and. We denote by $H$ the mean curvature vector, that is

$$
H(p)=\frac{1}{n} \sum_{i=1}^{n} h\left(e_{i}, e_{i}\right) .
$$

$N$ is minimal if $H$ vanishes identically. We also set

$$
\|h\|^{2}=\sum_{i, j=1}^{n} g\left(h\left(e_{i}, e_{j}\right), h\left(e_{i}, e_{j}\right)\right) .
$$

Let $K\left(e_{i} \wedge e_{j}\right), 1 \leq i, j \leq n$, denote the sectional curvature of the plane section spanned by $e_{i}$ and $e_{j}$. Then the scalar curvature of $N$ is given by

$$
\tau=\sum_{i<j} K\left(e_{i} \wedge e_{j}\right)
$$

For a differentiable function $f$ on $N$, the Laplacian $\Delta f$ of $f$ is defined as

$$
\Delta f=\sum_{j=1}^{n}\left\{e_{j}\left(e_{j} f\right)-\nabla_{e_{j}} e_{j} f\right\} .
$$

A submanifold $N$ tangent to $\xi$ is said to be invariant (resp. anti-invariant) submanifold if $\phi\left(T_{p} N\right) \subset T_{p} N, \forall p \in N\left(\operatorname{resp} . \phi\left(T_{p} N\right) \subset T_{p}^{\perp} N, \forall p \in N\right)$.

Lemma 2.1. [5] Let $a_{1}, \ldots ., a_{n}$ be $n$ real numbers and let $k$ be an integer in $[2, n-1]$. Then for any partition $\left(n_{1}, \ldots, n_{k}\right)$ of $n$, we have

$$
\begin{gathered}
\sum_{1 \leq i_{1}<j_{1} \leq n_{1}} a_{i_{1}} a_{j_{1}}+\sum_{n_{1}+1 \leq i_{2}<j_{2} \leq n_{1}+n_{2}} a_{i_{2}} a_{j_{2}}+\ldots+\sum_{n_{1}+\ldots+n_{k-1}+1 \leq i_{k}<j_{k} \leq n} a_{i_{k}} a_{j_{k}} \\
\geq \frac{1}{2 k}\left[\left(a_{1}+\ldots+a_{n}\right)^{2}-k\left(a_{1}^{2}+\ldots+a_{n}^{2}\right)\right]
\end{gathered}
$$

with the equality holding if and only if $a_{1}+\ldots+a_{n_{1}}=\ldots . .=a_{n_{1}+\ldots .+n_{k-1}+1}+$ $\ldots+a_{n}$.

\section{Multiply CR-WARPED PRODUCT SUBMANifolds of GENERALized}

\section{SASAKIAN SPACE FORM}

In 2008, Chen and Dillen [6] studied an interesting inequality between the warping functions $\sigma_{2}, \ldots, \sigma_{k}$ and the squared norm of the second fundamental form $\|h\|^{2}$ of a multiply CR-warped product submanifold $N=N_{T} \times_{\sigma_{2}} N_{2} \times \ldots \times_{\sigma_{k}} N_{k}$ in a general Kaehler manifold. Later in 2010, A Olteanu [10] established the same inequality in case of a multiply CR-warped product submanifolds in a Kenmotsu 
space form. In the present article we achieve the inequality for multiply CR-warped product submanifolds in a generalized Sasakian space form.

From the decomposition (2.5) we may write

$$
h(X, Y)=h_{\phi D^{\perp}}(X, Y)+h_{\lambda}(X, Y) .
$$

Also it is known that for multiply CR-warped submanifold of a Riemannian manifold we have

$$
\nabla_{X} Z=\sum_{a=2}^{k}\left(X\left(\log \sigma_{a}\right)\right) Z^{a}
$$

for any vector fields $X$ in $D$ and $Z$ in $D^{\perp}$, where $Z^{a}$ denotes the $N_{a}$-component of $Z$.

For proving the main inequality we need the following lemmas.

Lemma 3.1. Let $N=N_{T} \times_{\sigma_{2}} N_{2} \times \ldots \times_{\sigma_{k}} N_{k}$ be a multiply CR-warped product submanifold of a generalized Sasakian space form $\bar{M}$. Then we have

(i) $h_{\phi D^{\perp}}(\phi X, Z)=\sum_{a=2}^{k}\left(X\left(\log \sigma_{a}\right)\right) \phi Z^{a}+\phi P_{Z} \phi X$,

(ii) $g\left(Q_{Z} X, \phi W\right)=g\left(P_{Z} \phi X, W\right)$,

(iii) $g(h(\phi X, Z), \phi h(X, Z))=\left\|h_{\lambda}(X, Z)\right\|^{2}-g\left(Q_{X} Z, \phi h_{\lambda}(X, Z)\right)$

for any vector fields $X$ in $D$ and $Z, W$ in $D^{\perp}$, where $Z^{a}$ denotes the $N_{a^{-}}$ component of $Z$.

Proof. From Gauss formula we can write

$$
\nabla_{Z} \phi X+h(\phi X, Z)=P_{Z} X+Q_{Z} X+\phi \nabla_{Z} X+\phi h(Z, X)
$$

or

$$
\begin{gathered}
h(\phi X, Z)=P_{Z} X+Q_{Z} X+\phi\left(\sum_{a=2}^{k}\left(X\left(\log \sigma_{a}\right)\right) Z^{a}\right) \\
+\phi h(Z, X)-\sum_{a=2}^{k}\left(\phi X\left(\log \sigma_{a}\right)\right) Z^{a} .
\end{gathered}
$$

Comparing tangential parts in the above equation and then taking inner product with $W \in D^{\perp}$, we get

$$
h_{\phi D^{\perp}}(Z, X)=-\sum_{a=2}^{k}\left(\phi X\left(\log \sigma_{a}\right)\right) \phi Z^{a}+\phi P_{Z} X,
$$

which may be written as

$$
h_{\phi D^{\perp}}(\phi X, Z)=\sum_{a=2}^{k}\left(X\left(\log \sigma_{a}\right)\right) \phi Z^{a}+\phi P_{Z} \phi X, \quad \forall X \in D, Z \in D^{\perp} .
$$

This is part (i) of the lemma.

Now comparing normal parts of equation (3.2) we get

$$
h(\phi X, Z)-\phi h_{\lambda}(Z, X)=Q_{Z} X+\sum_{a=2}^{k}\left(X\left(\log \sigma_{a}\right) \phi Z^{a}\right)
$$


By taking inner product with $\phi W$ of the above equation we obtain

$$
g\left(h_{\phi D^{\perp}}(\phi X, Z), \phi W\right)=g\left(Q_{Z} X, \phi W\right)+\sum_{a=2}^{k}\left(X\left(\log \sigma_{a}\right) g\left(\phi Z^{a}, \phi W\right)\right) .
$$

Simplifying the above equation by using part (i) of the lemma we arrive at

$$
g\left(P_{Z} \phi X, W\right)=g\left(Q_{Z} X, \phi W\right)
$$

which proves part (ii).

Taking inner product of equation $(3.3)$ by $\phi h(X, Z)$ gives part (iii) of the lemma.

Theorem 3.1. Let $N=N_{T} \times_{\sigma_{2}} N_{2} \times \ldots \times{ }_{\sigma_{k}} N_{k}$ be multiply CR-warped product submanifold of a generalized Sasakian space form $\bar{M}$ with $P_{D^{\perp}} D \in D$, then the squared norm of the second fundamental form satisfies

$$
\|h\|^{2} \geq \sum_{a=2}^{k} n_{a}^{2}\left\|\left(\nabla \log \sigma_{a}\right)\right\|^{2}+\left\|P_{D^{\perp}} D\right\|^{2} .
$$

Proof. Let $\left\{X_{\circ}=\xi, X_{1}, X_{2}, \ldots, X_{p}, X_{p+1}=\phi X_{1}, \ldots, X_{2 p}=\phi X_{p}\right\}$ be a local orthonormal frame of vector fields on $N_{T}$ and $\left\{Z_{1}, Z_{2}, \ldots, Z_{q}\right\}$ be such that $Z_{\Delta_{a}}$ is a basis for some $N_{a}, a=2, \ldots, k$ where $\Delta_{2}=\left\{1,2, . ., n_{2}\right\}, \ldots ., \Delta_{k}=\left\{n_{2}+n_{3}+\ldots+\right.$ $\left.n_{k-1}+1, \ldots, n_{1}+n_{2}+\ldots+n_{k}\right\}$ and $n_{2}+n_{3}+\ldots .+n_{k}=q$. Then we have

$$
\begin{aligned}
\|h\|^{2}= & \sum_{i, j=1}^{2 p} g\left(h\left(X_{i}, X_{j}\right), h\left(X_{i}, X_{j}\right)\right)+\sum_{i=1}^{2 p} g\left(h\left(X_{i}, \xi\right), h\left(X_{i}, \xi\right)\right) \\
& +\sum_{i=1}^{2 p} \sum_{a=2}^{k} g\left(h\left(X_{i}, Z_{\Delta_{a}}\right), h\left(X_{i}, Z_{\Delta_{a}}\right)\right)+\sum_{a, b=2}^{k} g\left(h\left(Z_{\Delta_{a}}, Z_{\Delta_{b}}\right), h\left(Z_{\Delta_{a}}, Z_{\Delta_{b}}\right)\right) \\
& +\sum_{a=2}^{k} g\left(h\left(Z_{\Delta_{a}}, \xi\right), h\left(Z_{\Delta_{a}}, \xi\right)\right) .
\end{aligned}
$$

The above equation implies

$$
\|h\|^{2} \geq \sum_{i=1}^{2 p} \sum_{a=2}^{k} g\left(h\left(X_{i}, Z_{\Delta_{a}}\right), h\left(X_{i}, Z_{\Delta_{a}}\right)\right) .
$$

Now using part (i) of lemma-3.1 we get

$$
\|h\|^{2} \geq \sum_{i=1}^{2 p} \sum_{a=2}^{k} g\left(n_{a}\left(\phi X_{i}\left(\log \sigma_{a}\right)\right) \phi Z_{\Delta_{a}}+\phi P_{Z_{\Delta_{a}}} X_{i}, n_{a}\left(\phi X_{i}\left(\log \sigma_{a}\right)\right) \phi Z_{\Delta_{a}}+\phi P_{Z_{\Delta_{a}}} X_{i}\right) .
$$

In view of the assumption $P_{D^{\perp}} D \in D$, the above inequality takes the form

$$
\|h\|^{2} \geq \sum_{a=2}^{k} n_{a}^{2}\left\|\nabla \log \sigma_{a}\right\|^{2}\left\|Z_{\Delta_{a}}\right\|^{2}+\left\|P_{D^{\perp}} D\right\|^{2} .
$$

By Cauchy-Schwartz inequality the above equation becomes

$$
\sum_{a=2}^{k} n_{a}^{2}\left\|\nabla \log \sigma_{a}\right\|^{2}\left\|Z_{\Delta_{a}}\right\|^{2}+\left\|P_{D^{\perp}} D\right\|^{2} \geq \sum_{a=2}^{k} n_{a}^{2}\left\|\left(\nabla \log \sigma_{a}\right) Z_{\Delta_{a}}\right\|^{2}+\left\|P_{D^{\perp}} D\right\|^{2}
$$

Therefore 


$$
\|h\|^{2} \geq \sum_{a=2}^{k} n_{a}^{2}\left\|\left(\nabla \log \sigma_{a}\right)\right\|^{2}+\left\|P_{D^{\perp}} D\right\|^{2} .
$$

Hence we finish the proof.

We now prove the main inequality of this section

Theorem 3.2. Let $N=N_{T} \times_{\sigma_{2}} N_{2} \times \ldots \times_{\sigma_{k}} N_{k}$ be a multiply CR-warped product submanifold of a generalized Sasakian space form $\bar{M}$ with $P_{D^{\perp}} D \in D$, then

$$
\|h\|^{2} \geq \sum_{a=2}^{k}\left[n_{a}\left\{\left(\Delta \log \sigma_{a}\right)+n_{a}\left\|\nabla \log \sigma_{a}\right\|^{2}\right\} g\left(Z^{a}, Z^{a}\right)\right]+2 f_{2} p q .
$$

Proof. Let $X \in D$ and $Z \in D^{\perp}$, then from equation (2.1) we have

$$
\bar{R}(X, \phi X, Z, \phi Z)=-2 f_{2} g(X, X) g(Z, Z) .
$$

On the other hand from equation (2.3)

$$
\bar{R}(X, \phi X, Z, \phi Z)=g\left(\nabla_{X}^{\perp} h(\phi X, Z), \phi Z\right)-g\left(h\left(\nabla_{X} \phi X, Z\right), \phi Z\right)
$$

$$
-g\left(h\left(\phi X, \nabla_{X} Z\right), \phi Z\right)-g\left(\nabla_{\phi X}^{\perp} h(X, Z), \phi Z\right)+g\left(h\left(\nabla_{\phi X} X, Z\right), \phi Z\right)+g\left(h\left(X, \nabla_{\phi X} Z\right), \phi Z\right) \text {. }
$$

We will calculate each term of the above expression to get the required inequality. Now

$$
g\left(\nabla_{X}^{\perp} h(\phi X, Z), \phi Z\right)=X g(h(\phi X, Z), \phi Z)-g\left(h(\phi X, Z), \bar{\nabla}_{X} \phi Z\right) .
$$

Using equation (3.3) we get the value of first term of the above equation as

$$
X g(h(\phi X, Z), \phi Z)=X\left\{\sum_{a=2}^{k}\left(X\left(\log \sigma_{a}\right) g\left(Z^{a}, Z^{a}\right)\right)\right\}
$$

or equivalently

$$
X g(h(\phi X, Z), \phi Z)=\sum_{a=2}^{k}\left[\left\{X\left(X \log \sigma_{a}\right)+2\left(X \log \sigma_{a}\right)^{2}\right\} g\left(Z^{a}, Z^{a}\right)\right] .
$$

Also the second term in equation (3.6) is obtained as

$$
g\left(h(\phi X, Z), \bar{\nabla}_{X} \phi Z\right)=g\left(h(\phi X, Z),\left(\bar{\nabla}_{X} \phi\right) Z\right)+g\left(h(\phi X, Z), \phi\left(\bar{\nabla}_{X} Z\right)\right) .
$$

Using equation (3.1) and part-(iii) of lemma-3.1, it follows that

$$
\begin{aligned}
g\left(h(\phi X, Z), \bar{\nabla}_{X} \phi Z\right)= & g\left(h(\phi X, Z)-\phi h(X, Z), Q_{X} Z\right) \\
& +\sum_{a=2}^{k}\left(\left(X \log \sigma_{a}\right) g\left(h(\phi X, Z), \phi Z^{a}\right)\right)+\left\|h_{\lambda}(X, Z)\right\|^{2} .
\end{aligned}
$$

Now making use of equation (3.3) and part-(ii) of lemma-3.1 in the above equation we get finally

(3.8) $g\left(h(\phi X, Z), \bar{\nabla}_{X} \phi Z\right)=\sum_{a=2}^{k}\left(\left(X \log \sigma_{a}\right)^{2} g\left(Z^{a}, Z^{a}\right)\right)-\left\|Q_{X} Z\right\|^{2}+\left\|h_{\lambda}(X, Z)\right\|^{2}$. 
Putting equations (3.7) and (3.8) in equation (3.6) we have

$$
\begin{aligned}
g\left(\nabla_{X}^{\perp} h(\phi X, Z), \phi Z\right) & =\sum_{a=2}^{k}\left[\left\{X\left(X \log \sigma_{a}\right)+\left(X \log \sigma_{a}\right)^{2}\right\} g\left(Z^{a}, Z^{a}\right)\right] \\
& +\left\|Q_{X} Z\right\|^{2}-\left\|h_{\lambda}(X, Z)\right\|^{2} .
\end{aligned}
$$

Similarly we obtain

$$
\begin{aligned}
g\left(\nabla_{\phi X}^{\perp} h(\phi X, Z), \phi Z\right) & =\sum_{a=2}^{k}\left[\left\{\phi X\left(\phi X \log \sigma_{a}\right)+\left(\phi X \log \sigma_{a}\right)^{2}\right\} g\left(Z^{a}, Z^{a}\right)\right] \\
& +\left\|Q_{\phi X} Z\right\|^{2}-\left\|h_{\lambda}(X, Z)\right\|^{2} .
\end{aligned}
$$

Again using part-(i) of lemma-3.1 we evaluate

$$
g\left(h\left(\phi X, \nabla_{X} Z\right), \phi Z\right)=\sum_{a=2}^{k}\left\{\left(X \log \sigma_{a}\right)^{2} g\left(Z^{a}, Z^{a}\right)\right\} .
$$

Similarly we have

$$
g\left(h\left(X, \nabla_{X} Z\right), \phi Z\right)=-\sum_{a=2}^{k}\left\{\left(\phi X \log \sigma_{a}\right)^{2} g\left(Z^{a}, Z^{a}\right)\right\} .
$$

Using again part-(i) of lemma-3.1 we have

$$
g\left(h\left(\nabla_{\phi X} X, Z\right), \phi Z\right)=-\sum_{a=2}^{k}\left\{\left(\phi \nabla_{\phi X} X \log \sigma_{a}\right)^{2} g\left(Z^{a}, Z^{a}\right)\right\} .
$$

Similarly we have

$$
g\left(h\left(\nabla_{X} \phi X, Z\right), \phi Z\right)=-\sum_{a=2}^{k}\left\{\left(\phi \nabla_{X} \phi X \log \sigma_{a}\right)^{2} g\left(Z^{a}, Z^{a}\right)\right\} .
$$

The last equation may further be simplified by using the fact that $N_{T}$ is totally geodesic in $N$, equation (3.1) and Gauss equation as

$$
\begin{gathered}
g\left(h\left(\nabla_{X} \phi X, Z\right), \phi Z\right)=\sum_{a=2}^{k}\left\{\left(\nabla_{X} X \log \sigma_{a}\right) g\left(Z^{a}, Z^{a}\right)\right\} \\
+\sum_{a=2}^{k}\left\{\left(\nabla_{\phi X} \phi X \log \sigma_{a}\right) g\left(Z^{a}, Z^{a}\right)\right\}-\sum_{a=2}^{k}\left\{\left(\phi \nabla_{\phi X} X \log \sigma_{a}\right) g\left(Z^{a}, Z^{a}\right)\right\} .
\end{gathered}
$$

Substituting equations (3.9)-(3.14) into equation (3.5) it becomes

$$
\begin{gathered}
\bar{R}(X, \phi X, Z, \phi Z)=\sum_{a=2}^{k}\left\{\left(X\left(X \log \sigma_{a}\right)+\phi X\left(\phi X \log \sigma_{a}\right)-\nabla_{X} X \log \sigma_{a}-\nabla_{\phi X} \phi X \log \sigma_{a}\right) g\left(Z^{a}, Z^{a}\right)\right\} \\
+\left\|Q_{X} Z\right\|^{2}+\left\|Q_{\phi X} Z\right\|^{2}-\left\|h_{\lambda}(X, Z)\right\|^{2}-\left\|h_{\lambda}(\phi X, Z)\right\|^{2} .
\end{gathered}
$$

Putting the bases of $N_{T}, N_{2}, \ldots N_{k}$ and summing over them we find

$$
\sum_{i=1}^{p} \sum_{\Delta_{a}} \bar{R}\left(X_{i}, \phi X_{i}, Z_{\Delta_{a}}, \phi Z_{\Delta_{a}}\right)=\sum_{a=2}^{k} n_{a}\left(\Delta \log \sigma_{a}\right) g\left(Z^{a}, Z^{a}\right)
$$




$$
+\left\|Q_{D} D^{\perp}\right\|^{2}-\left\|h_{\lambda}\left(D, D^{\perp}\right)\right\|^{2} .
$$

On the other hand equation (3.4) gives

$$
\sum_{i=1}^{p} \sum_{\Delta_{a}} \bar{R}\left(X_{i}, \phi X_{i}, Z_{\Delta_{a}}, \phi Z_{\Delta_{a}}\right)=-2 f_{2} p q
$$

Combining equations (3.15) and (3.16) we get

$$
\left\|h_{\lambda}\left(D, D^{\perp}\right)\right\|^{2}=\sum_{a=2}^{k} n_{a}\left(\Delta \log \sigma_{a}\right) g\left(Z^{a}, Z^{a}\right)+\left\|Q_{D} D^{\perp}\right\|^{2}+2 f_{2} p q .
$$

It is easy to see from part-(i) of lemma-3.1 that

$$
\left\|h_{\phi D^{\perp}}\left(D, D^{\perp}\right)\right\|^{2}=\sum_{a=2}^{k} n_{a}^{2}\left\|\nabla \log \sigma_{a}\right\|^{2} g\left(Z^{a}, Z^{a}\right)+\left\|P_{D^{\perp}} D\right\|^{2} .
$$

Therefore equations (3.17) and (3.18) give

$$
\begin{aligned}
\|h\|^{2}= & \sum_{a=2}^{k} n_{a}\left(\Delta \log \sigma_{a}\right) g\left(Z^{a}, Z^{a}\right)+\left\|Q_{D} D^{\perp}\right\|^{2}+2 f_{2} p q \\
& +\sum_{a=2}^{k} n_{a}^{2}\left\|\nabla \log \sigma_{a}\right\|^{2} g\left(Z^{a}, Z^{a}\right)+\left\|P_{D^{\perp}} D\right\|^{2},
\end{aligned}
$$

which proves that

$$
\|h\|^{2} \geq \sum_{a=2}^{k}\left[n_{a}\left\{\left(\Delta \log \sigma_{a}\right)+n_{a}\left\|\nabla \log \sigma_{a}\right\|^{2}\right\} g\left(Z^{a}, Z^{a}\right)\right]+2 f_{2} p q .
$$

\section{ANTi-INVARiant MUltiply WARPED PRODUCT SUBMANifolds OF A GENERALIZED SASAKIAN SPACE FORM}

In 2008 Chen and Dillen [6] obtained a sharp inequality for warping functions $\sigma_{2}, \ldots, \sigma_{k}$ of a multiply warped product submanifold $N=N_{1} \times_{\sigma_{2}} N_{2} \times \ldots \times{ }_{\sigma_{k}} N_{k}$ isometrically immersed in an arbitrary Riemannian manifold and the squared mean curvature $\|H\|^{2}$. Later in 2010, A. Olteanu [10] established the similar inequality in case of anti-invariant submanifold of a Kenmotsu space form. In this paper we achieve the same type of inequality for anti-invariant submanifold of a Generalized Sasakian space form.

We know from [10] that the following relation holds for warping functions

$$
\Delta \sigma_{a}=\sigma_{a} \sum_{j=1}^{n_{1}} K\left(e_{j} \wedge X_{a}\right)
$$

for any unit vector tangent $X_{a}$ tangent to $N_{a}$, where $\left\{e_{1}, e_{2}, \ldots, e_{n_{1}}\right\}$ is an orthonormal basis of $T_{\pi_{1}(p)} N_{1}$. 


\section{Case I- When $\xi$ is tangent to $N$.}

Theorem 4.1. Let $\psi$ be an anti-invariant isometric immersion of an $n$-dimensional multiply warped $N=N_{1} \times_{\sigma_{2}} N_{2} \times \ldots \times{ }_{\sigma_{k}} N_{k}$ into a $(2 m+1)$-dimensional generalized Sasakian space form $\bar{M}$ with $\xi$ tangent to $N$. Then

$$
\sum_{a=2}^{k} n_{a} \frac{\Delta \sigma_{a}}{\sigma_{a}} \leq n_{1}\left(n-n_{1}\right) f_{1}-f_{3}+\frac{n^{2}}{2}\left(1-\frac{1}{k}\right)\|H\|^{2} .
$$

Proof. Let $N=N_{1} \times{ }_{\sigma_{2}} N_{2} \times \ldots \times{ }_{\sigma_{k}} N_{k}$ be an anti-invariant multiply warped product submanifold in a generalized Sasakian space form $\bar{M}$. Then from equation (2.1) and (2.2) the sectional curvature $K_{N}(X \wedge Y)$ determined by orthonormal vectors $X$ and $Y$ is given by

(4.2) $K_{N}(X \wedge Y)=f_{1}-f_{3}\left\{\eta(X)^{2}+\eta(Y)^{2}\right\}+g(h(X, X), h(Y, Y))-\|h(X, Y)\|^{2}$.

Since $\xi$ is tangent to $N$, using equation (2.7) the above equation becomes

$$
\sum_{i \neq j} K_{N}\left(e_{i} \wedge e_{j}\right)=2 \tau=n(n-1) f_{1}-2 f_{3}+n^{2}\|H\|^{2}-\|h\|^{2} .
$$

We put [6]

$$
\eta=2 \tau-n^{2}\|H\|^{2}-n(n-1) f_{1}+2 f_{3}+\frac{n^{2}}{k}\|H\|^{2}
$$

and hence we have

$$
n^{2}\|H\|^{2}=k\left(\eta+\|h\|^{2}\right) .
$$

We choose an orthonormal basis $e_{1}, e_{2}, \ldots, e_{2 m+1}$ at $p$ such that, for each $j \in \Delta_{a}$, $e_{j}$ is tangent to $N_{a}$ for $a=1, \ldots, k$. Moreover we choose the normal vector field $e_{n+1}$ in the direction of the mean curvature vector at $p$. Therefore the above equation yields

$$
\frac{1}{2 k}\left[\left(\sum_{A=1}^{n} a_{A}\right)^{2}-k \sum_{A=1}^{n} a_{A}^{2}\right]=\frac{1}{2}\left[\eta+\sum_{A \neq B}\left(h_{A B}^{n+1}\right)^{2}+\sum_{r=n+2}^{2 m+1} \sum_{A, B=1}^{n}\left(h_{A B}^{r}\right)^{2}\right] .
$$

Now applying basic lemma-2.1 in the above equation we get

$$
\begin{gathered}
\sum_{\alpha_{1}<\beta_{1}} a_{\alpha_{1}} a_{\beta_{1}}+\sum_{\alpha_{2}<\beta_{2}} a_{\alpha_{2}} a_{\beta_{2}}+\ldots+\sum_{\alpha_{k}<\beta_{k}} a_{\alpha_{k}} a_{\beta_{k}} \geq \\
\frac{\eta}{2}+\sum_{A<B}\left(h_{A B}^{n+1}\right)^{2}+\frac{1}{2} \sum_{r=n+2}^{2 m+1} \sum_{A, B=1}^{n}\left(h_{A B}^{r}\right)^{2} .
\end{gathered}
$$

Now using equation (4.1) we may write

$$
\begin{aligned}
& \sum_{a=2}^{k} n_{a} \frac{\Delta \sigma_{a}}{\sigma_{a}}=\sum_{j \in \Delta_{1}} \sum_{\beta \in \Delta_{2} \cup \ldots \cup \Delta_{k}} K\left(e_{j} \wedge e_{\beta}\right)+\sum_{1 \leq j_{1}<j_{2} \leq n_{1}} K\left(e_{j_{1}} \wedge e_{j_{2}}\right) \\
&+\sum_{n_{1}+1 \leq \alpha<\beta \leq n} K\left(e_{\alpha} \wedge e_{\beta}\right)-\sum_{1 \leq j_{1}<j_{2} \leq n_{1}} K\left(e_{j_{1}} \wedge e_{j_{2}}\right)-\sum_{n_{1}+1 \leq \alpha<\beta \leq n} K\left(e_{\alpha} \wedge e_{\beta}\right),
\end{aligned}
$$

from which we have

$$
\sum_{a=2}^{k} n_{a} \frac{\Delta \sigma_{a}}{\sigma_{a}} \leq \tau-\frac{n(n-1)}{2} f_{1}+n_{1}\left(n-n_{1}\right) f_{1}-\frac{\eta}{2}
$$


By putting the value of $\eta$ from equation (4.2) in the above equation we obtain the required inequality.

We note easily the following corollaries from the theorem as obtained in [9]-

Corollary 4.1. Let $N=N_{1} \times_{\sigma_{2}} N_{2} \times \ldots \times_{\sigma_{k}} N_{k}$ be a multiply warped product into a generalized Sasakian space form $\bar{M}$ such that $N=N_{1} \times_{\sigma_{2}} N_{2} \times \ldots \times{ }_{\sigma_{k}} N_{k}$ is an anti-invariant submanifold tangent to $\xi$. If the warping functions $\sigma_{a}, a=2, \ldots, k$ are harmonic, then $N=N_{1} \times_{\sigma_{2}} N_{2} \times \ldots \times_{\sigma_{k}} N_{k}$ admits no minimal immersion into a generalized Sasakian space form $\bar{M}$ with $f_{1}<0$ and $f_{3}>0$.

Corollary 4.2. If the warping functions $\sigma_{a}, a=2, \ldots, k$ of an anti-invariant multiply warped product submanifold $N=N_{1} \times{ }_{\sigma_{2}} N_{2} \times \ldots \times{ }_{\sigma_{k}} N_{k}$ tangent to $\xi$ in a generalized Sasakian space form $\bar{M}$ are eigen functions of the Laplacian with corresponding eigenvalues $\lambda_{a}>0, a=2, \ldots, k$ respectively, then $N=N_{1} \times_{\sigma_{2}} N_{2} \times \ldots \times{ }_{\sigma_{k}} N_{k}$ admits no minimal immersion in a generalized Sasakian space form $\bar{M}$ with $f_{1} \leq 0$ and $f_{3} \geq 0$.

Corollary 4.3. Let $N=N_{1} \times{ }_{\sigma_{2}} N_{2} \times \ldots \times{ }_{\sigma_{k}} N_{k}$ be an anti-invariant multiply warped product submanifold tangent to $\xi$ in a generalized Sasakian space form $\bar{M}$. If $\sigma_{a}$, $a=2, . ., k_{1}$ are harmonic and $\sigma_{b}, b=1, \ldots, k_{2}$ are eigen functions of the Laplacian with corresponding eigenvalues $\lambda_{b}, b=1, . ., k_{2}$ respectively, where $k_{1}+k_{2}=k$, then $N=N_{1} \times{ }_{\sigma_{2}} N_{2} \times \ldots \times_{\sigma_{k}} N_{k}$ admits no minimal immersion into a generalized Sasakian space form $\bar{M}$ with $f_{1} \leq 0$ and $f_{3} \geq 0$.

\section{Case-II When $\xi$ is normal to $N$.}

Theorem 4.2. Let $\psi$ be an anti-invariant isometric immersion of an $n$-dimensional multiply warped $N=N_{1} \times{ }_{\sigma_{2}} N_{2} \times \ldots \times{ }_{\sigma_{k}} N_{k}$ into a $(2 m+1)$-dimensional generalized Sasakian space form $\bar{M}$ with $\xi$ normal to $N$. Then

$$
\sum_{a=2}^{k} n_{a} \frac{\Delta \sigma_{a}}{\sigma_{a}} \leq n_{1}\left(n-n_{1}\right) f_{1}+\frac{n^{2}}{2}\left(1-\frac{1}{k}\right)\|H\|^{2} .
$$

Proof. Since $\xi$ is normal to $N$ we have from equation (4.2)

$$
\sum_{i, j} K_{N}\left(e_{i} \wedge e_{j}\right)=2 \tau=n(n-1) f_{1}+n^{2}\|H\|^{2}-\|h\|^{2} .
$$

Then proceed as in case-I, we obtain the final inequality as

$$
\sum_{a=2}^{k} n_{a} \frac{\Delta \sigma_{a}}{\sigma_{a}} \leq n_{1}\left(n-n_{1}\right) f_{1}+\frac{n^{2}}{2}\left(1-\frac{1}{k}\right)\|H\|^{2} .
$$

Hence the theorem is proved.

We extract the following straight corollaries from this theorem-

Corollary 4.4. Let $N=N_{1} \times_{\sigma_{2}} N_{2} \times \ldots \times_{\sigma_{k}} N_{k}$ be a multiply warped product into a generalized Sasakian space form $\bar{M}$ such that $N=N_{1} \times_{\sigma_{2}} N_{2} \times \ldots \times \times_{\sigma_{k}} N_{k}$ is an anti-invariant submanifold normal to $\xi$. If the warping functions $\sigma_{a}, a=2, \ldots, k$ are harmonic, then $N=N_{1} \times{ }_{\sigma_{2}} N_{2} \times \ldots \times{ }_{\sigma_{k}} N_{k}$ admits no minimal immersion into a generalized Sasakian space form $\bar{M}$ with $f_{1}<0$. 
Corollary 4.5. If the warping functions $\sigma_{a}, a=2, \ldots, k$ of an anti-invariant multiply warped product submanifold $N=N_{1} \times_{\sigma_{2}} N_{2} \times \ldots \times{ }_{\sigma_{k}} N_{k}$ normal to $\xi$ in a generalized Sasakian space form $\bar{M}$ are eigen functions of the Laplacian with corresponding eigenvalues $\lambda_{a}>0, a=2, \ldots, k$ respectively, then $N=N_{1} \times{ }_{\sigma_{2}} N_{2} \times \ldots \times{ }_{\sigma_{k}} N_{k}$ admits no minimal immersion in a generalized Sasakian space form $\bar{M}$ with $f_{1} \leq 0$.

Corollary 4.6. Let $N=N_{1} \times{ }_{\sigma_{2}} N_{2} \times \ldots \times{ }_{\sigma_{k}} N_{k}$ be an anti-invariant multiply warped product submanifold normal to $\xi$ in a generalized Sasakian space form $\bar{M}$. If $\sigma_{a}$, $a=2, . ., k_{1}$ are harmonic and $\sigma_{b}, b=1, \ldots, k_{2}$ are eigen functions of the Laplacian with corresponding eigenvalues $\lambda_{b}, b=1, . ., k_{2}$ respectively, where $k_{1}+k_{2}=k$, then $N=N_{1} \times_{\sigma_{2}} N_{2} \times \ldots \times_{\sigma_{k}} N_{k}$ admits no minimal immersion into a generalized Sasakian space form $\bar{M}$ with $f_{1} \leq 0$.

\section{REFERENCES}

[1] Alegre, P., Blair, D. E. and Carriazo, A., Generalized Sasakian space forms, Israel J. Math., 141(2004), 157-183.

[2] Atceken, M., Warped product semi-slant submanifolds in Kenmotsu manifolds, Turk. J. Math., 34(2010), 425-433.

[3] Chen, B. Y., Geometry of warped product CR-submanifolds in Kaehler manifold, Monatsh. Math., 133(2001), 177-195.

[4] Chen, B. Y., Geometry of warped product CR-submanifolds in Kaehler manifold II, Monatsh. Math., 134(2001), 103-119.

[5] Chen, B. Y., Ricci curvature of real hypersurfaces in complex hyperbolic space, Arch. Math. (Brno) 38(2002), 73-80.

[6] Chen, B. Y. and Dillen, F., Optimal inequalities for multiply warped product submanifolds, Int. Electron. J. Geom., 1(2008), 1-11.

[7] Mihai, I., Contact CR-warped product submanifolds in Sasakian space forms, Geom. Dedicata, 109(2004), 165-173.

[8] Munteanu, M. I., Warped product contact CR-submanifolds of Sasakian space form, Publ. Math. Debrecen, 66(6)(2005), 75-120.

[9] Olteanu, A., A general inequality for doubly warped product submanifolds, Math. J. Okayama Univ., 52(2010), 133-142.

[10] Olteanu, A., Multiply warped product submanifolds in Kenmotsu space forms, Bull. Inst. Math. Acad. Sinica(New Series), 5(2010), No-2, 201-214.

[11] Sahin, B., Non-existence of warped product semi-slant submanifolds of Kaehler manifold, Geom. Dedicata, 117(2006), 195-202.

Department of Mathematics, Jamia Millia Islamia, New Delhi-25-India

E-mail address: jamalidbdyahoo@gmail.com and hasan_jmi@yahoo.com 